Article

\title{
Evaluation of In-Season Management Zones from High-Resolution Soil and Plant Sensors
}

\author{
Martina Corti ${ }^{1, *}$, Pietro Marino Gallina ${ }^{1}$ (D), Daniele Cavalli ${ }^{1}$, Bianca Ortuani ${ }^{1}$, \\ Giovanni Cabassi ${ }^{2}$, Gabriele Cola ${ }^{1}\left(\mathbb{D}\right.$, Antonio Vigoni ${ }^{3}$, Luigi Degano ${ }^{2}$ \\ and Simone Bregaglio 4 (D) \\ 1 Department of Agricultural and Environmental Sciences-Production, Landscape, Agroenergy, \\ Università degli Studi di Milano, via Celoria 2, 20133 Milano, Italy; pietro.marino@unimi.it (P.M.G.); \\ daniele.cavalli@unimi.it (D.C.); bianca.ortuani@unimi.it (B.O.); gab.cola@gmail.com (G.C.) \\ 2 CREA-Council for Agricultural Research and Economics, Research Centre for Animal Production and \\ Aquaculture, via Antonio Lombardo 11, 26900 Lodi, Italy; giovanni.cabassi@crea.gov.it (G.C.); \\ luigi.degano@crea.gov.it (L.D.) \\ 3 Sport Turf Consulting-Servizi per l'Agricoltura con Aeromobili a Pilotaggio Remoto, Via Cesare Battisti, 19, \\ 20027 Rescaldina, MI, Italy; stc@turfgrass.it \\ 4 CREA-Council for Agricultural Research and Economics, Research Centre for Agriculture and Environment, \\ via di Corticella 133, 40128 Bologna, Italy; simoneugomaria.bregaglio@crea.gov.it \\ * Correspondence: martina.corti@unimi.it
}

Received: 12 June 2020; Accepted: 28 July 2020; Published: 3 August 2020

check for updates

\begin{abstract}
The adoption of precision agriculture has the potential to increase the environmental sustainability of cropping systems as well as farmers' income. Farmers in transition to precision agriculture need low-input and effective protocols to delineate homogenous management zones to optimize their actions without past knowledge e.g., yield maps. Different approaches have been developed so far, based on the analysis of the within-field variability in crop and soil properties, but procedures were rarely suited for operational conditions. We identified here a low-inputs protocol to map management zones from soil electrical conductivity and/or crop vegetation indices, using a winter wheat field in northern Italy as a pilot case. The reliability of the alternative data sources was evaluated at three crop development stages using a yield map as reference. Red-edge and NIR (NDRE) bands were the most reliable data sources for management zones identification, with $62 \%$, $68 \%$, and $74 \%$ of correct classifications at early tillering, stem elongation, and late booting, respectively. Our work identifies a minimum dataset for accurate management zones' definition and highlights that in-season monitoring based on the red-edge band was able to reliably identify management zones already at early tillering, despite minor differences in crop growth.
\end{abstract}

Keywords: UAV; EMI sensor; management zones definition; within-field spatial variability; normalized difference vegetation indices; soil apparent electrical conductivity; yield map

\section{Introduction}

The intensification of agricultural systems led to the excessive use of irrigation water, fertilizers, and other agrochemicals, with detrimental effects on environmental sustainability and farmers' profitability [1]. Standard management practices in conventional agriculture entail consistent application of water and agrochemicals, disregarding the within-field variability of crop status and needs [2]. Precision agriculture (PA) aims at improving the resources use efficiency to maximize yield and reduce production costs, through the optimization of the timing and the supply of agrochemicals [3]. The assessment of the within-field variability of soil and vegetation properties is the basis of PA because it allows modulating farmer inputs release according to the actual crop requirements [2]. A key concept 
in PA is the variable-rate application, based either on application maps or on real-time sensors [4]. Examples of the latter are tractor-mounted data acquisition and processing systems used to optimize nitrogen fertilization [5,6] and weed management [7].

Map-based techniques are used to define management zones (MZs) characterized by homogeneous yield-limiting factors [8], allowing a site-specific application of agrochemicals [9]. The first methodology used in MZs definition consisted of manual samplings to determine soil properties and crop yield [9-11]. This approach was replaced by proximal sensors to measure apparent soil electrical conductivity (ECa), a proxy of multiple physical and chemical soil properties [12]. Then, the multispectral response of vegetation acquired by satellites or by aerial vehicles has been progressively adopted in MZs definition [8]. After the soil and/or crop sensing has been performed, statistical analyses are used [13] to integrate soil properties and spectral crop data $[8,14,15]$. The most used statistical methods are k-means clustering, fuzzy clustering, neural network models, classification trees, and principal components analysis [16,17]. All these methods have been used with different input variables, including soil and crop properties [18] as well as crop yield [19], without any reference approach or data source widely recognized [20]. Moreover, the timing of field surveys and the number of MZs are often set according to the specific MZs mapping method, rather than driven by agronomic indications [17]. Therefore, there is the need of identifying effective and low-input procedures for MZs mapping among the plethora of existing methodologies and data sources, especially in contexts where farmers are transitioning to precision agriculture, with no ex ante knowledge of the within-field variability. This is the case of wheat systems in northern Italy, where the adoption of precision agriculture has just begun despite its possible benefits in safeguarding environmental suitability while maximizing crop yields.

This study aims at defining a low-input methodology for MZs mapping based on high-resolution soil and crop sensors (EMI sensor and airborne digital camera), which can be adopted by farmers in the first year of adoption of precision agriculture when yield maps are not available. Alternative sources of information recorded during the wheat growing season were evaluated by multivariate data analysis and then used for MZs mapping. The resulting maps were analyzed to identify the most useful period and data source for the field survey to optimize wheat management. The performances of the different methods of MZs mapping were evaluated according to their accuracy, reliability, and discrimination ability in reproducing actual yield. The agronomic meaning of the resulting MZs was finally validated via an experimental trial set up to study the crop response to different nitrogen levels in each MZ.

\section{Materials and Methods}

Figure 1 shows the workflow of our study, which is articulated in three steps: soil, vegetation, and yield data acquisition and processing (Section 2.2), and data analysis, where (i) multivariate analysis of the different sources was performed, (ii) MZs map derived by different data sources were evaluated, and (iii) the experimental field data were analyzed (Section 2.3).

A soil survey was performed before winter wheat sowing with an electromagnetic multi-frequency electromagnetic induction (EMI) sensor to measure soil electrical conductivity, considered as a proxy for texture and organic matter (Section 2.2.1). Then, vegetation monitoring has been carried out in three wheat development stages (early tillering, $\mathrm{BBCH} 25$; beginning of stem elongation, $\mathrm{BBCH} 31$; late booting stage, $\mathrm{BBCH} 45$ ) [21], using a multispectral camera mounted on a unmanned aerial vehicle (UAV), and simple and multi-temporal vegetation indices were calculated (Section 2.2.2). At harvest, a yield map was acquired (Section 2.2.4) and used as a reference to evaluate the accuracy of the alternative sources for MZs mapping.

Multivariate analysis was performed to highlight the contribution of soil and vegetation data on MZs definition (Section 2.3.1) and to select the best predictors of yield. Then, alternative MZs were drawn via k-means fuzzy clustering (Section 2.3.2). These maps were compared with the reference yield map via contingency tables (Section 2.3.3). 
In order to evaluate the agronomical value of the identified MZs, a field trial (Section 2.3.4) was carried out (Section 2.2.3), to study the impact of MZs together with nitrogen fertilization levels on crop growth.

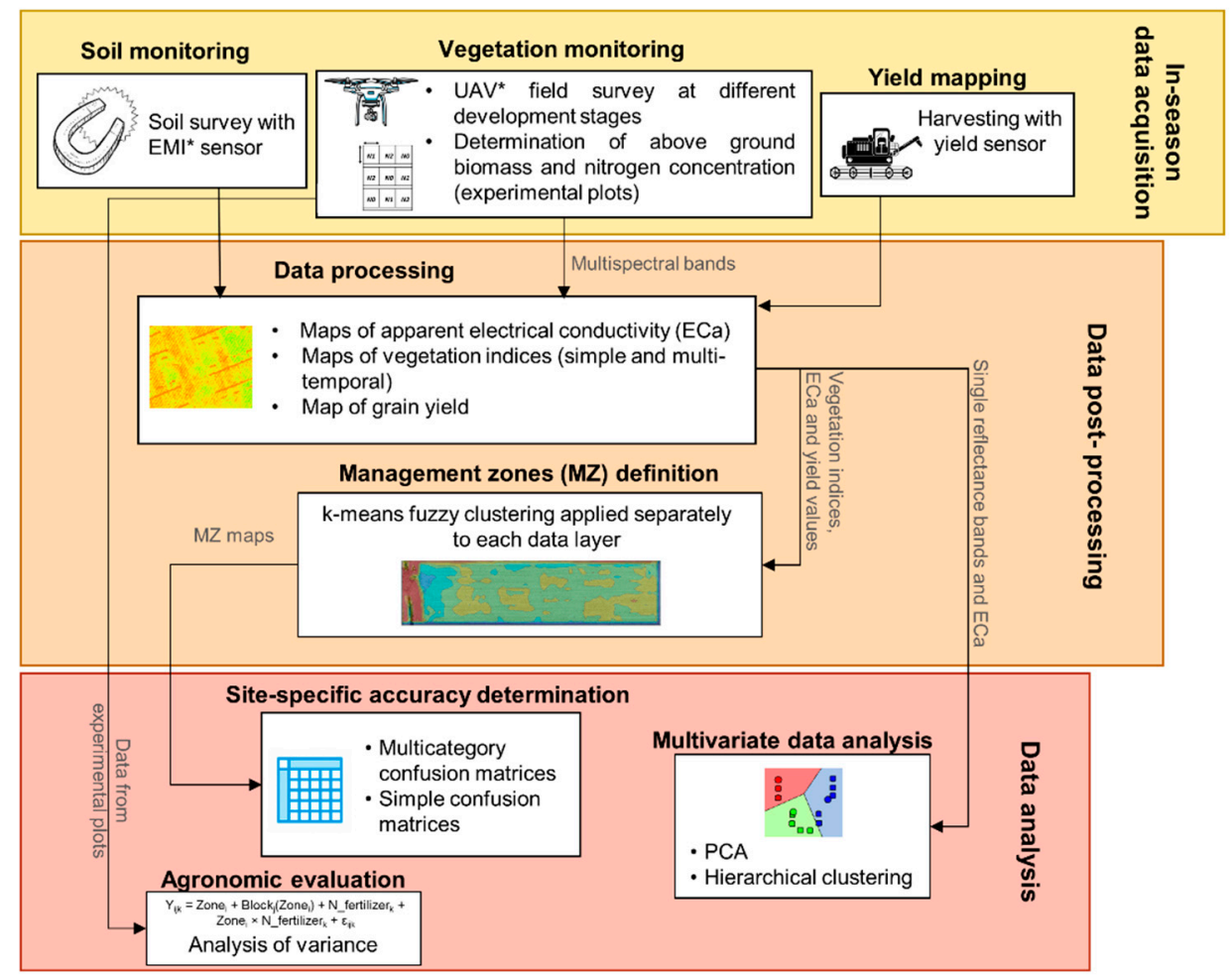

Figure 1. Schematic representation of the activities carried out in the study. ${ }^{*} \mathrm{EMI}=$ electromagnetic induction; $\mathrm{UAV}=$ unmanned aerial vehicle; ECa: apparent electrical conductivity; MZ: management zone.

\subsection{Site Description and Crop Management}

The MZs mapping was performed in a winter wheat (Triticum aestivum, var. Basmati) field of 11.3 ha located in Sant'Angelo Lodigiano (Lodi), Italy $\left(45^{\circ} 13^{\prime} 19.6644^{\prime \prime} \mathrm{N}, 9^{\circ} 25^{\prime} 42.7548^{\prime \prime}\right.$ E, altitude $73 \mathrm{~m}$ asl) at Cascina Belfuggito, owned by Morando Bolognini Foundation. Wheat was sown on 10th November 2015, fertilizations were performed on 13th February 2016 (ammonium nitrate, $52 \mathrm{~kg} \mathrm{~N} \mathrm{ha}^{-1}$ ), and on 18th March 2016 (urea, $92 \mathrm{~kg} \mathrm{~N} \mathrm{ha}^{-1}$ ); wheat was harvested on 28th June 2016.

Minimum and maximum daily air temperatures in 2015-2016 growing season were in line with the climatic norm (1993-2016, Figure S1), and were not limiting for wheat development. Precipitations in the early crop establishment phase were lower than the average (November-January: $29 \mathrm{~mm}$ vs. $213 \mathrm{~mm}$ ), whereas February, May, and June were characterized by larger precipitations than the averages $(145,121$, and $71 \mathrm{~mm}$ vs. 51,77 , and $54 \mathrm{~mm})$, leading to cumulated values similar to the climatic norm (441 mm vs. $523 \mathrm{~mm}$ ) (Figure S2).

\subsection{In-Season Data Acquisition and Processing}

\subsubsection{Soil Monitoring and Mapping}

The geophysical sensor Profiler EMP400 (Geophysical Survey Systems, Inc., Nashua, NH, USA) was used to measure soil properties. This sensor is based on electromagnetic induction. It measures ECa at a frequency ranging from 1 to $16 \mathrm{kHz}$, to explore increasing soil depths. The ECa measurements taken at $15 \mathrm{kHz}$ were considered here, with a vertical orientation of dipoles, corresponding to about $1 \mathrm{~m}$ of soil depth of exploration, according to maximum root depth of winter wheat. The survey was carried out at the end of January 2016, when the soil water content was close to field capacity. 
The ECa measurements were interpolated by ordinary kriging (Figure S4) using the software GStat [22]. The experimental variogram was calculated, and the parameters of the variogram model were selected and validated. All the ECa data were interpolated on a $1 \mathrm{~m}$ grid, then resampled at $5 \mathrm{~m}$ to allow comparing soil MZs maps with the other maps.

\subsubsection{Monitoring and Mapping the Within-Field Crop Variability}

Two surveys were carried out with a multispectral camera (MicaSense RedEdge digital camera; MicaSense, Inc., Seattle, WA, USA) mounted on a UAV to acquire crop data to characterize the crop spatial variability. The multispectral camera acquires reflectance in five discrete bands: blue band (B), centered at $475 \mathrm{~nm}$; green band (G), centered at $560 \mathrm{~nm}$; red band (R), centered at $668 \mathrm{~nm}$; red-edge band (RE), centered at $717 \mathrm{~nm}$; near-infrared band (NIR), centered at $840 \mathrm{~nm}$. The camera was mounted on a coaxial octocopter UAV platform, with a maximum take-off mass of $12 \mathrm{~kg}$ and equipped with a GNSS (Global Navigation Satellite System) NEO-M8N (u-blox, Thalwil, Switzerland).

Image acquisition was carried out in three wheat development stages: early tillering (eTl), beginning of stem elongation (StEl), and late boot stage (1B). The field surveys were performed at $120 \mathrm{~m}$ altitude at low flight speed $\left(8 \mathrm{~m} \mathrm{~s}^{-1}\right)$. The flight plan guaranteed $80 \%$ of forward and sideward overlapped, as needed for the mosaicking process. Before and after each flight, images of a white reference panel of known reflectance were acquired, allowing the radiometric calibration of the images collected by the camera. All the images were uploaded to the MicaSense Atlas cloud, a service for data pre-processing and production of orthoimage, i.e., a geo-referenced single 5-band 16-bit image containing the reflectance values expressed as digital numbers. The orthoimage had a spatial resolution of $8.23 \mathrm{~cm}$, then it was resampled at $5 \mathrm{~m}$ grid using the QGIS software (version 3.4, Madeira). The grid dimension of $5 \mathrm{~m}$, was chosen as a compromise between the very high resolution of the digital cameras used and the operative grid of agricultural machinery used for field operations. Moreover, the $5 \mathrm{~m}$ grid allowed processing data in a time suitable for operational condition.

Different normalized difference vegetation indices (Table 1) were calculated using QGIS software (version 3.4, Madeira) from the multispectral data, to obtain the vegetation maps used for MZs definition. Three indices were considered: the normalized difference vegetation index (NDVI), the green normalized difference vegetation index (GNDVI), and the normalized difference red-edge index (NDRE). The NDVI is the most widely used index to map crop vigor. The GNDVI and NDRE are known to better reflect the leaf chlorophyll content than NDVI, which is more affected by canopy structural changes. Finally, the cumulative vegetation indices [23] were calculated to test the impact of subsequent surveys on MZs mapping performances.

Table 1. Definition, acronyms, and equations of the vegetation indices used in the study. Cumulative vegetation index (cumVI) was calculated for each vegetation index present in the table (VI) at three wheat development stages $(1=$ early tillering, $2=$ stem elongation, and $3=$ late booting).

\begin{tabular}{lcc}
\hline \multicolumn{1}{c}{ Definition } & Vegetation Index & Equation \\
\hline Normalized difference vegetation index & NDVI & $(\mathrm{NIR}-\mathrm{R}) /(\mathrm{NIR}+\mathrm{R})$ \\
Green normalized difference vegetation index & GNDVI & $(\mathrm{NIR}-\mathrm{G}) /(\mathrm{NIR}+\mathrm{G})$ \\
Normalized difference red-edge & $\mathrm{NDRE}$ & $(\mathrm{NIR}-\mathrm{B}) /(\mathrm{NIR}+\mathrm{B})$ \\
Cumulative vegetation index & cumVI & $\sqrt{\mathrm{VI}_{1}^{2}+\mathrm{VI}_{2}^{2}+\mathrm{VI}_{3}^{2}}$ \\
\hline
\end{tabular}

\subsubsection{Monitoring Crop Growth Conditions: Crop Response to Nitrogen}

Crop response to $\mathrm{N}$ fertilization was evaluated in three zones within the field (Figure S3), by setting up a completely randomized block design with three replicates and three fertilization levels $(0,72$, and $144 \mathrm{~kg} \mathrm{ha}^{-1}$, identified as N0, N1, and N2, respectively). The three zones were selected according to the preliminary analysis of soil ECa map (Figure S4) and visual assessment of Google aerial images. Fertilizers were hand-spread on the experimental plots $(5 \times 7 \mathrm{~m}): 36 \%$ of the total rate was applied as 
ammonium nitrate on 13th February 2016 (BBCH 23) and the remaining 64\% was applied as urea on 18th March 2016 (BBCH 25). Wheat plants grown in $0.5 \mathrm{~m}^{2}$ were cut above the collar in correspondence to the UAV-surveys and at harvest, to determine aboveground biomass (AGB) dry matter (DM) $\left(\mathrm{t} \mathrm{DM} \mathrm{ha}{ }^{-1}\right)$ and $\mathrm{N}$ uptake $\left(\mathrm{kg} \mathrm{N} \mathrm{ha}^{-1}\right)$. The samples were oven-dried at $105^{\circ} \mathrm{C}$ to determine dry weight and ground with a rotary-knife mill equipped with a sieve of $4 \mathrm{~mm}$ mesh; subsequently, a part was ground with a ZM 100 centrifugal mill equipped with a sieve of $0.2 \mathrm{~mm}$ mesh (Retsch Gmbh \& Co., Haan, Germany). The total $\mathrm{N}$ of samples was determined by dry combustion using ThermoQuest NA1500 elemental analyzer (Carlo Erba).

\subsubsection{Yield Monitoring}

The crop was harvested on 28th June 2016 with a Claas Lexion 570 (CLAAS KGaA mbH, Harsewinkel, Germany) harvester equipped with sensors for yield mapping. Harvested grains were collected in a wagon and weighted, to correct the yield data acquired through the harvester.

The yield map consisted of a data layer containing the geographical coordinates of points and the associated yield measurements from the harvester sensor. The vector of points was transformed into a vector of polygons by the Voronoi tessellation implemented in QGIS, and the resulting map was then resampled at a $5 \mathrm{~m}$ grid.

\subsection{Data Analysis}

\subsubsection{Multivariate Data Analysis}

Principal component analysis (PCA) was performed to identify patterns of similarity in the spatial variability of soil and crop data and to identify the most meaningful source of information. The input variables of PCA were the five bands recorded at each UAV flight (B, R, G, RE, and NIR bands) and the ECa values all with the same weight; yield data, development stages, and Y-MZs were not used to compute principal components (PCs) but their coordinates in the PCs space were calculated after the analysis (supplementary variables). An agglomerative hierarchical clustering on PCs was then performed (Euclidean metrics and Ward's minimum variance method were set to build the tree). Each cluster was described by the mean values of the original variables (ECa, B, R, $\mathrm{RE}$, and NIR bands). Moreover, each cluster was qualitatively described taking into account both spatial and temporal variability, i.e., labeling pixels depending on wheat development stages and the three yield-based MZs (low, medium, and high productivity). For each supplementary variable, a v-test was calculated to quantify its presence in the clusters [24]. The sign of the v-statistic indicated over- and under-representation (positive and negative, respectively) of the category in the cluster. The PCA and hierarchical clustering on PCs were performed using FactoMineR R package [24] in R 3.6.2 environment [25].

\subsubsection{Management Zones Definition}

Management zone analyst software [26] was used to perform k-means fuzzy clustering to identify MZs. The k-means algorithm is based on the minimization of the sum of squared Euclidean distances of all data points from the cluster barycenter, through an iterative process that assigns data points to clusters until a minimum error is reached. The following clustering parameters were specified: maximum number of iterations $=300$, convergence criterion $=0.0001$, minimum number of clusters $=2$, maximum number of clusters $=4$. Fuzzy k-means further uses a weighting exponent to control partial memberships to the cluster, which was set to 1.3 [27]. The optimal number of classes was chosen according to the default method from the software. The results of MZA clustering were the predictor-based MZs maps, based on soil or crop properties: ECa-based MZs, indicated as soil-MZ; NDVI-, NDRE-, and GNDVI-based MZs in the three development stages, indicated as [VI]-MZ, where VI is the vegetation index (e.g., NDVI-MZs). The yield-based MZs map (Y-MZs) was produced by MZA clustering and considered as the reference map. 


\subsubsection{Site-Specific Accuracy Assessment of the Delineated MZs}

The similarity of each predictor-based MZs map with the Y-MZs map was evaluated via contingency tables [28]. Two statistics were calculated: the proportion of correct classification (PCC), i.e., the number of grid cells correctly assigned to the different MZs divided by the total number of cells, and the Heidke skill scores (HSS), which measures the accuracy of the classification without considering the fraction of cells which would be correctly classified due to random chance (Equation (1)) [28]:

$$
H S S=\frac{\sum_{i=1}^{I} p\left(p r M Z_{i}, y M Z_{i)-} \sum_{i=1}^{I} p\left(p r M Z_{i}\right) p\left(y M Z_{i}\right)\right.}{1-\sum_{i=1}^{I} p\left(p r M Z_{i}\right) p\left(y M Z_{i}\right)} \times 100,
$$

where $p\left(p r M Z_{i}, y M Z_{i}\right)$ is the joint distribution of predictor-based MZs and $Y-M Z s, p\left(p r M Z_{i}\right)$ is the marginal distribution of predictor-based $M Z s$ classification, and $p\left(y M Z_{i}\right)$ is the marginal distribution of Y-MZs classification, for each of the I couples of predictor-based MZs and yield-based MZs classes.

Each multi-category contingency table, resulting from the comparison between the different MZs maps and Y-MZs, was reduced to dichotomous tables (Figure 2).

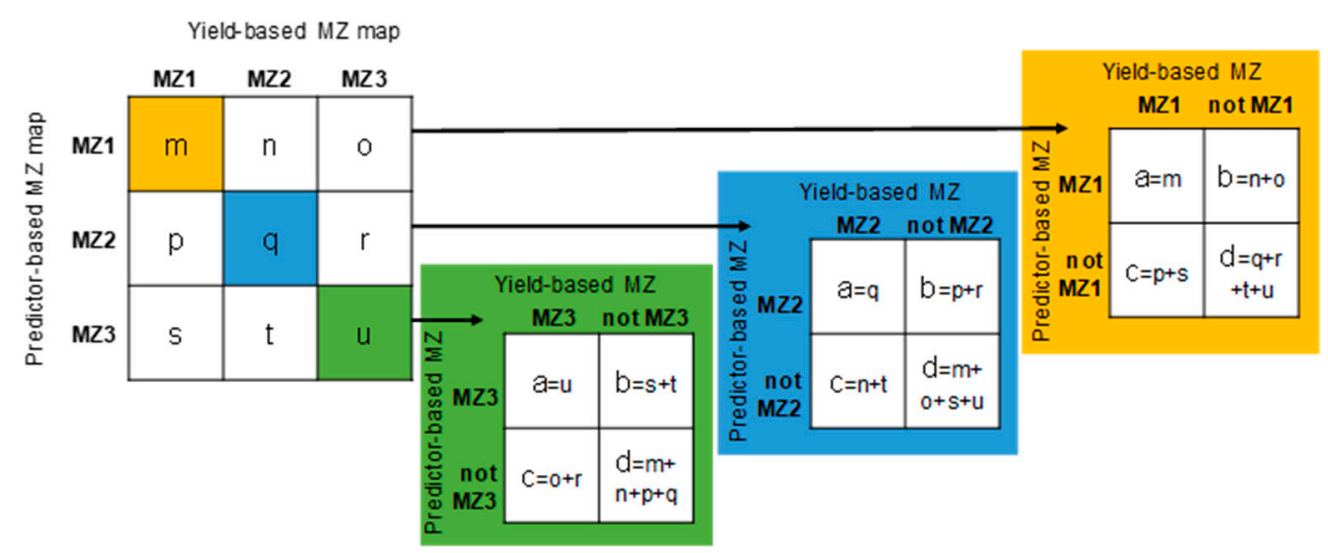

Figure 2. Example of 3-classes contingency matrix and its reduction to one table for each class (dichotomous tables).

Six statistics for each MZs map were calculated from the dichotomous tables (for the definition of $a, b, c, d$, refer to Figure 2): (1) Critical success index $($ CSI $=a /(a+b+c)$, with optimum at 1$)$, the proportion of correct classifications after removing incorrect classifications; (2) Bias (BIAS = $(\mathrm{a}+$ $\mathrm{b}) /(\mathrm{a}+\mathrm{c})$, with optimum at 1$)$, the ratio of the predictor-based $M \mathrm{Z}_{\mathrm{i}}$ and the $\mathrm{Y}-\mathrm{MZ}_{\mathrm{i}}$; (3) False alarm ratio $(F A R=b /(a+b)$, with optimum at 0$)$, the proportion of predictor-based $M Z_{i}$ that did not correspond to the same Y-MZi; (4) Probability of false detection $(\mathrm{F}=\mathrm{b} /(\mathrm{b}+\mathrm{d})$, with optimum at 0$)$, the ratio of false alarms in reproducing $\mathrm{Y}-\mathrm{MZ}_{\mathrm{i}} ;(5)$ Hit rate $(\mathrm{H}=\mathrm{a} /(\mathrm{a}+\mathrm{c})$, with optimum at 1$)$, the ratio of correctly classified MZ; (6) Proportion of correct classification $(P C C=(a+d) /(a+b+c+d)$, with optimum at 1$)$. The contingency tables were analyzed with MATLAB (version 2014b; MathWorks, Natick, MA, USA).

\subsubsection{Agronomic Evaluation}

The accompany plot trial was designed in order to understand the causes of variability of crop growth and yield in the MZs during the whole growing season. The effects of the zone and $\mathrm{N}$ fertilization on wheat yield and $\mathrm{N}$ uptake were assessed by a split-plot ANOVA model. The between-subject factor "zone" was considered fixed with three levels (correspondent to the yield-based Y-MZ1, Y-MZ2, and Y-MZ3); subjects (blocks) within each zone were random, and the within-subject factor " $\mathrm{N}$ fertilizer" was fixed with three levels $\left(0,72\right.$, and $\left.144 \mathrm{~kg} \mathrm{~N} \mathrm{ha}^{-1}\right)$. Planned orthogonal contrasts were used to assess the linear and quadratic trends in AGB and $\mathrm{N}$ uptake in response to $\mathrm{N}$ fertilizer and to assess the differences between zones (zone 1 vs. zone 2 and 3, and zone 2 vs. zone 3 ) as well as the interaction 
between factors. ANOVA was executed with the GLM procedure in SPSS Version 26.0.0.1 (IBM Inc., Armonk, NY; contrasts were calculated with the LMATRIX and MMATRIX commands. The effects of factors and their interactions were considered significant at $p=0.05$.

\section{Results}

\subsection{Multivariate Data Analysis}

The results of PCA and hierarchical clustering applied to the principal components are shown in Figure 3.

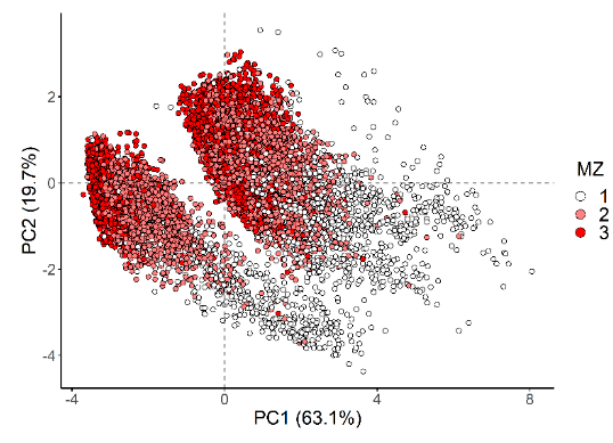

(a)

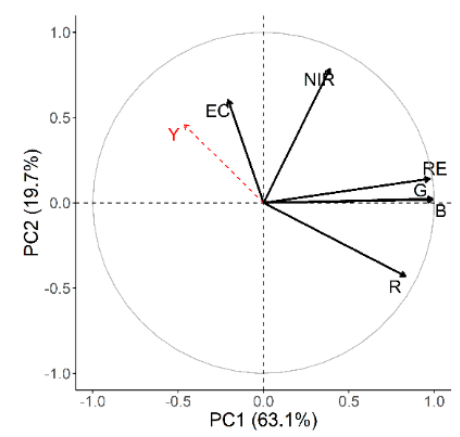

(c)

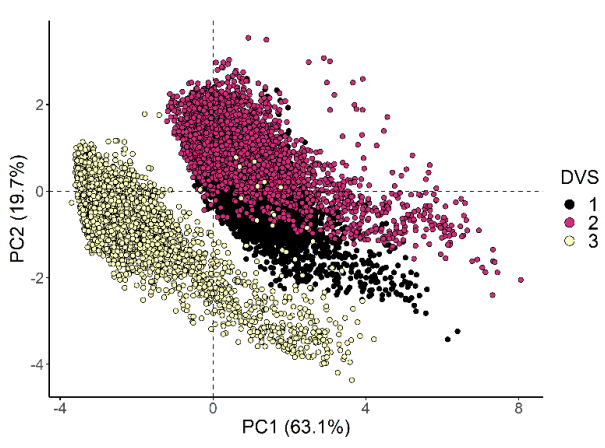

(b)

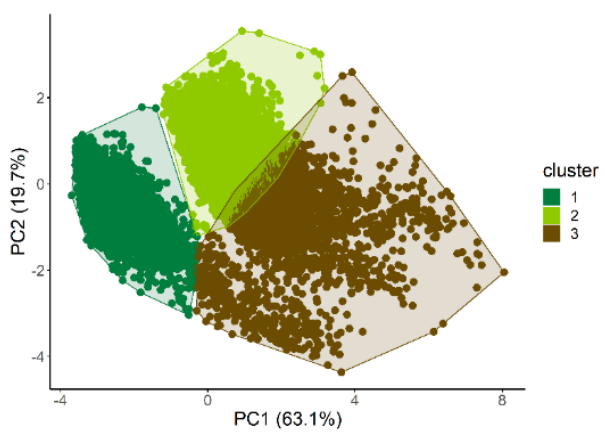

(d)

Figure 3. Results of principal component analysis (PCA). PCA score plots of individuals (pixels) showing the pixels in the first two principal components space marked according to (a) the yield-based management zones, and (b) the development stages at the time of crop monitoring (DVS: 1, 2, and 3 corresponded to eTl, StEl, and 1B). (c) PCA plot of the original variables projected into the first two principal components space. (d) PC score-plot showing the three extracted clusters.

The first two principal components (PC) accounted for $83 \%$ of the total variance and were selected to summarize the data. The PC1 explained $63 \%$ of the total variance and had a strong positive correlation with B, G, R, RE bands $(r>0.95, p<0.05)$. As shown in Figure $3 b$, spectral crop properties at eTl and StEl were similar, whereas at $\mathrm{IB}$, generally higher reflectance in the five bands was recorded. The second principal component (PC2) explained $20 \%$ of the total variance and was positively correlated with NIR $(r=0.78, p<0.05)$ and with ECa $(r=0.60, p<0.05)$ and negatively correlated to the R band $(r=-0.43, p<0.05)$. As shown in Figure 3a, PC1 and PC2 show a gradient of separation of pixels belonging to Y-MZ1 from pixels in Y-MZ2 and Y-MZ3. Yield values had a strong negative correlation with crop reflectance in the $\mathrm{R}$ band $(r=-0.57, p<0.05)$ and positive correlation with the ECa and NIR band ( $r=0.23$ and 0.21 , respectively, $p<0.05$ ). This suggested that Y-MZ1 could be identified by both ECa and by red-based vegetation indices with the NIR component. The angle between yield and NIR suggested that indices with the NIR component could be able to identify different yield levels within 
the field, irrespective of the visible band chosen (Figure 3c). The visible bands B, G, and RE carried the same information, indeed.

The results of the hierarchical clustering are presented in Figure $3 \mathrm{~d}$, while the description of clusters through qualitative and quantitative variables are reported in Tables S1 and S2. The main difference between clusters 1 and 2 is the wheat development stages at sampling time. Data in cluster 1 were characterized by the lowest reflectance values in all the collected bands, corresponding to $\mathrm{BB}$ field surveys (100\% of the data in cluster 1 were collected at $1 \mathrm{~B})$ and to high yield MZs (Table S1). Cluster 2 was mainly composed of data acquired at the StEl stage (60\%). This cluster was characterized by high reflectance values in the NIR and RE bands (Figure 3c,d), corresponding to high vegetation. Cluster 3 was mainly composed of data belonging to Y-MZ1 (low yield, $44 \%)$, with $64 \%(p<0.05)$ of data collected at eTL. Spectral data collected in Y-MZ1 were characterized by high reflectance values of visible bands, in particular of $R$ band, suggesting a stunted crop growth. Cluster 3 was characterized by high values in the visible bands and low values in the NIR band, leading to a clear distinction from the other clusters already at early wheat development. Furthermore, the mean ECa value in cluster 3 was $1.8 \mathrm{mS} \mathrm{m}^{-1}$, very similar to the mean value of reference Y-MZ1 $\left(1.1 \mathrm{mS} \mathrm{m}^{-1}\right)$, much lower compared to the average ECa of the field $\left(2.8 \mathrm{mS} \mathrm{m}^{-1}\right.$, Table S2).

\subsection{MZs Maps}

Figure 4 shows the results of MZs mapping with different data sources, i.e., yield, NDVI at different development stages, cumulative NDVI, and ECa. The NDVI values are used as representative of the other vegetation indices since the results were very similar.

The Y-MZs map identified three MZs: Y-MZ1 was characterized by lower wheat yield (average $4.5 \mathrm{t} \mathrm{DM} \mathrm{ha}^{-1}$ ) than Y-MZ2 and Y-MZ3, which produced on average 7.0 and $9.0 \mathrm{t} \mathrm{DM} \mathrm{ha}^{-1}$, respectively. The mean value of ECa was very variable (CV in the range $45-105 \%$ ) and mainly differed in Y-MZ1 vs. Y-MZ2 and Y-MZ3 (mean values: 1.7 vs. $1.9 \mathrm{mS} \mathrm{m}^{-1}$ ), while the NDVI, averaged across surveys, increased from MZ1 (0.76) to MZ2 (0.87) and MZ3 (0.93), indicating marked variability of crop vigor within the field. The three soil-MZs based on the ECa map were characterized by very different ECa values (average 1.1, 2.8, and $4.7 \mathrm{mS} \mathrm{m}^{-1}$ for MZ1-3, respectively) and large CV. However, the yield variability among soil-MZs was much lower than in the Y-MZs map, ranging from $6.8 \mathrm{t} \mathrm{DM} \mathrm{ha}{ }^{-1}$ in soil-MZ1 to $7.8 \mathrm{t} \mathrm{DM} \mathrm{ha}^{-1}$ in soil-MZ2 and soil-MZ3. Moreover, the average NDVI along wheat development in soil-MZ1 (0.87) was slightly lower than in soil-MZ2 and soil-MZ3, which obtained the same value (0.90). The NDVI-MZs at different development stages were more similar to the Y-MZs, both considering yield values and their spatial variability (Figure 4). At lB, the NDVI-MZ1 had the lowest NDVI (0.68) with the largest variability $(\mathrm{CV}=10 \%)$ and led to lower yield $\left(4.4 \mathrm{t} \mathrm{DM} \mathrm{ha}{ }^{-1}\right)$ compared to NDVI-MZ2 (6.7 t DM ha ${ }^{-1}$ ) and NDVI-MZ3 $\left(8.4 \mathrm{t} \mathrm{DM} \mathrm{ha}^{-1}\right)$. The NDVI values in NDVI-MZ2 slightly decreased after StEl (mean NDVI were $0.85,0.90$, and 0.87 at eTl, StEl, and 1B, respectively), indicating some limitation to wheat growth. The extension of NDVI-MZ3 decreased during wheat development, and the corresponding values of the NDVI values were high and constant (0.90, 0.94, and 0.94 at eTl, StEl, and $\mathrm{lB}$ stages, respectively). The cumulative vegetation index (cumNDVI) was calculated as a single index summarizing the temporal variation of each pixel. The cumNDVI increased according to yield (mean cumNDVI was 1.3, 1.5, and 1.6 in cumNDVI-MZ1, cumNDVI-MZ2, and cumNDVI-MZ3, respectively). However, the area of NDVI-MZ2 increased from StEl to lB stages (2.3 vs. $3.1 \mathrm{ha}$ ), and the area of NDVI-MZ3 decreased (6.9 vs. $5.7 \mathrm{ha}$ ), in agreement with the Y-MZs map, while the extension of cumNDVI-MZ3 was overestimated (6.3 ha vs. 4.1 ha of yield map), probably because cumNDVI did not allow discriminating changes in crop vigor. 

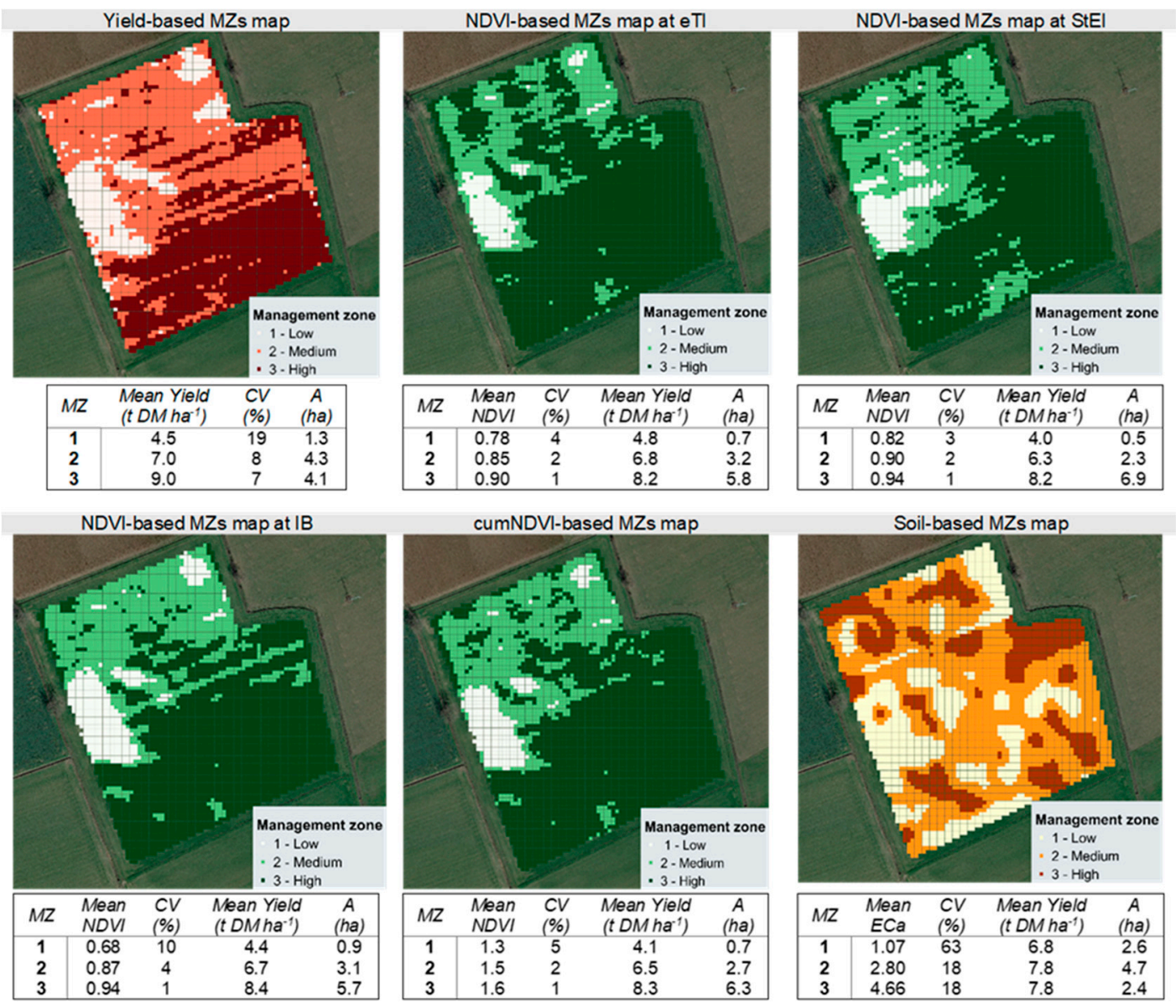

Figure 4. Management zone (MZ) maps resulting from yield data; NDVI measured at early tillering stage (eTl), stem elongation stage (StEl), late booting stage (lB); cumulative NDVI (cumNDVI); ECa. For each map and MZ, the mean value and the coefficient of variation $(\mathrm{CV})$ of the predictor is reported, as well as the average yield and the covered area.

\subsection{Site-Specific Accuracy of MZs Mapping}

A graphical representation of the accuracy of MZs mapping is presented in Figure 5, and the evaluation metrics are reported in Table 2. Vegetation indices-based maps showed the highest agreement with the Y-MZs map (Figure 5), particularly for the indices based on the G and RE bands (GNDVI and NDRE, respectively), which obtained the highest PCC and HSS scores (Figure 5).

At eTl, GNDVI- and NDRE-MZs maps misclassified grain yield areas in $11 \%$ and $19 \%$ of the total number of pixels, respectively. On the contrary, the NDVI-MZs map led to a constant yield overestimation throughout the growing season, with $32 \%$ (eTl) and $35 \%$ (StEl, lB) of pixels leading to 1 or 2 classes mismatch with respect to the Y-MZs map, mostly localized in Y-MZ2. All the vegetation indices obtained better performances at increasing development stages (PCC $=63 \%, 72 \%$, and 74\%; HSS $=39 \%, 54 \%$, and $57 \%$ for NDVI, GNDVI, and NDRE, respectively). Nonetheless, the overestimation of high yield areas increased along with wheat development stages. These errors were concentrated in the transition areas among MZs. Soil-MZs map was characterized by the lowest PCC (43\%) and HSS $(11 \%)$ and by a large number of pixels corresponding to yield underestimation ( $41 \%)$, associated to 1 class $(9 \%)$ or 2 classes (32\%) misclassification. The highest misclassification levels belonged to areas of the field with the highest yield.

The soil-MZs map was characterized by higher accuracy in identifying Y-MZ1 than Y-MZ2 and Y-MZ3 (H value $=0.62$ ), even with overestimation (BIAS $=1.94$ ) in $60 \%$ of the pixels (Table 2$)$. Th three vegetation indices NDVI, GNDVI, and NDRE led to similar performances in reproducing the Y-MZs 
map, with higher accuracy (CSI and PCC), reliability (FAR), and discrimination ability (H and F) at increasing development stages (Table 2). At the $1 \mathrm{~B}$ stage, the percentage of correct $\mathrm{MZ}$ attribution was high for all indices (PCC $>0.65$, CSI $>0.48$ ), whereas NDVI-MZs map failed to identify Y-MZ2 $(\mathrm{CSI}=0.31)$ at the StEl stage. Coherently, FAR values decreased during the growing season, indicating lower errors towards crop maturity. The accuracy of the soil and vegetation maps was high in Y-MZ1 (i.e., lowest yield) even at early crop stages (Figure 5, Table 2). The NDVI had lower performances than the other indices, especially considering FAR ( 0.38 vs. 0.48 and 0.49 for GNDVI and NDRE, respectively) and $\mathrm{F}$ ( 0.03 vs. 0.08 and 0.10 for GNDVI and NDRE, respectively), except at the eTL stage. Moreover, NDVI had the highest BIAS, relative to the MZ3 at late development stages (1.68), probably due to a saturation effect. The NDRE had slightly better performances than GNDVI in MZs definition, with higher discrimination ability. The Y-MZ2 (medium yield) had the highest misclassification rate across soil and vegetation indices.

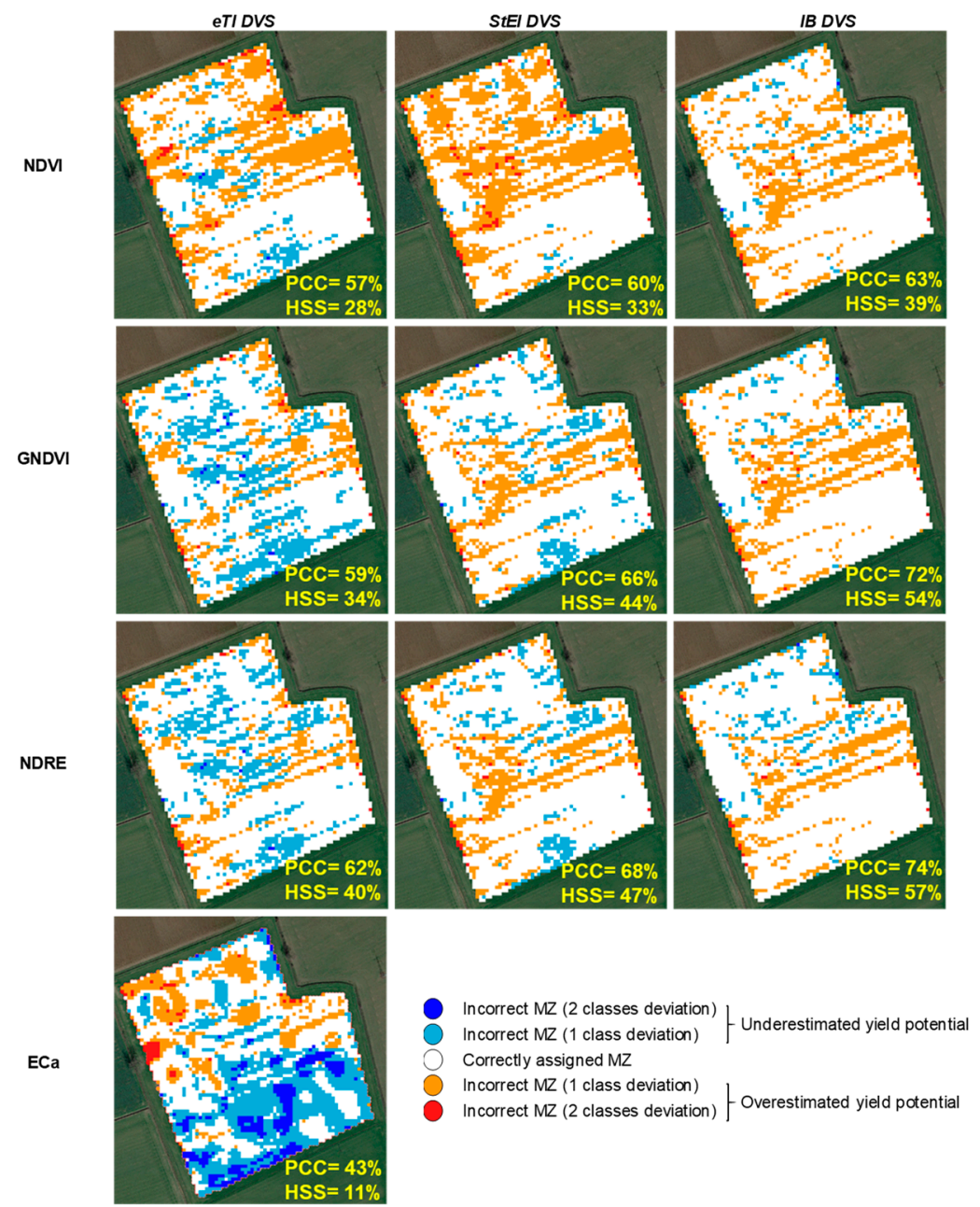

Figure 5. Graphical representation of the differences between the reference yield-based MZs map and the MZs maps based on vegetation indices acquired at three different development stages (NDVI, GNDVI, and NDRE), and ECa. For each map, the proportion of correct classification (PCC) MZs attribution, and the Heidke skill score (HSS) are reported. 
Table 2. Critical success index (CSI), BIAS, false alarm ratio (FAR), hit rate (H), probability of false detection (F), and the proportion of correct classification (PCC) were calculated separately for soil and vegetation indices, for MZs and development stage (before planting; early tillering, eTl; stem elongation, StEl; late booting, lB).

\begin{tabular}{|c|c|c|c|c|c|c|c|c|}
\hline INDEX & MZ & STAGE & CSI & BIAS & FAR & $\mathbf{H}$ & $\mathbf{F}$ & PCC \\
\hline \multirow{3}{*}{ ECA } & 1 & \multirow{3}{*}{ Before Planting } & 0.27 & 1.94 & 0.68 & 0.62 & 0.21 & 0.77 \\
\hline & 2 & & 0.34 & 1.11 & 0.52 & 0.53 & 0.47 & 0.53 \\
\hline & 3 & & 0.19 & 0.57 & 0.56 & 0.25 & 0.22 & 0.56 \\
\hline \multirow{9}{*}{ NDVI } & \multirow{3}{*}{1} & eTl & 0.28 & 0.54 & 0.38 & 0.33 & 0.03 & 0.88 \\
\hline & & StEl & 0.37 & 0.47 & 0.16 & 0.40 & 0.01 & 0.91 \\
\hline & & $1 \mathrm{~B}$ & 0.49 & 0.59 & 0.11 & 0.53 & 0.01 & 0.93 \\
\hline & \multirow{3}{*}{2} & eTl & 0.33 & 0.76 & 0.43 & 0.43 & 0.27 & 0.60 \\
\hline & & StEl & 0.31 & 0.61 & 0.38 & 0.38 & 0.19 & 0.62 \\
\hline & & lB & 0.31 & 0.51 & 0.29 & 0.36 & 0.12 & 0.65 \\
\hline & \multirow{3}{*}{3} & eTl & 0.49 & 1.42 & 0.44 & 0.80 & 0.43 & 0.66 \\
\hline & & StEl & 0.53 & 1.60 & 0.44 & 0.90 & 0.49 & 0.67 \\
\hline & & lB & 0.56 & 1.68 & 0.43 & 0.96 & 0.50 & 0.69 \\
\hline \multirow{9}{*}{ GNDVI } & \multirow{3}{*}{1} & eTl & 0.36 & 1.04 & 0.48 & 0.54 & 0.08 & 0.87 \\
\hline & & StEl & 0.50 & 0.81 & 0.25 & 0.60 & 0.03 & 0.92 \\
\hline & & lB & 0.56 & 0.77 & 0.17 & 0.64 & 0.02 & 0.93 \\
\hline & \multirow{3}{*}{2} & eTl & 0.39 & 0.92 & 0.42 & 0.54 & 0.32 & 0.62 \\
\hline & & StEl & 0.43 & 0.81 & 0.33 & 0.54 & 0.22 & 0.67 \\
\hline & & lB & 0.48 & 0.72 & 0.22 & 0.56 & 0.13 & 0.73 \\
\hline & \multirow{3}{*}{3} & eTl & 0.48 & 1.07 & 0.37 & 0.68 & 0.27 & 0.70 \\
\hline & & StEl & 0.56 & 1.27 & 0.36 & 0.81 & 0.32 & 0.74 \\
\hline & & lB & 0.63 & 1.38 & 0.33 & 0.92 & 0.32 & 0.78 \\
\hline \multirow{9}{*}{ NDRE } & \multirow{3}{*}{1} & eTl & 0.41 & 1.31 & 0.49 & 0.67 & 0.10 & 0.87 \\
\hline & & StEl & 0.52 & 0.91 & 0.28 & 0.65 & 0.04 & 0.92 \\
\hline & & 1B & 0.57 & 0.93 & 0.25 & 0.70 & 0.04 & 0.93 \\
\hline & \multirow{3}{*}{2} & eTl & 0.43 & 1.00 & 0.40 & 0.60 & 0.32 & 0.64 \\
\hline & & StEl & 0.46 & 0.87 & 0.32 & 0.59 & 0.23 & 0.69 \\
\hline & & lB & 0.52 & 0.78 & 0.22 & 0.61 & 0.14 & 0.75 \\
\hline & \multirow{3}{*}{3} & eTl & 0.50 & 0.90 & 0.29 & 0.64 & 0.18 & 0.74 \\
\hline & & StEl & 0.56 & 1.18 & 0.33 & 0.78 & 0.27 & 0.75 \\
\hline & & $1 \mathrm{~B}$ & 0.65 & 1.26 & 0.30 & 0.89 & 0.26 & 0.80 \\
\hline
\end{tabular}

Statistics in bold font are discussed in the main text.

\subsection{Agronomic Evaluation}

The areas of the field dedicated to the experimental plots were representative of the identified Y-MZs. The field trial showed that the response of AGB and $\mathrm{N}$ uptake to $\mathrm{N}$ fertilization, when averaged across the three zones, was positive and significant $(p<0.05)$ at all development stages (Figure 6 and Figure S5; Tables S3 and S4).

Starting from StEl, AGB and N uptake averaged across $\mathrm{N}$ levels were significantly lower in Y-MZ1 than in the other zones $(p<0.05)$. Finally, from booting onwards, the interaction between $\mathrm{N}$ level and management zone was significant $(p<0.05)$. The response of both dependent variables (AGB and $\mathrm{N}$ uptake) to $\mathrm{N}$ fertilizer was more flat in Y-MZ1 compared to the other zones. Differences between Y-MZ2 and Y-MZ3 emerged only at harvest, when N uptake in Y-MZ3 was significantly higher $(p<0.05)$. Therefore, average AGB at harvest in Y-MZ1 was about 70\% less than in the other zones ( 5 vs. $16 \mathrm{t} \mathrm{DM} \mathrm{ha}^{-1}$, respectively), while average $\mathrm{N}$ uptake was $73 \mathrm{~kg} \mathrm{~N} \mathrm{ha}^{-1}$ in Y-MZ1, $166 \mathrm{~kg} \mathrm{~N} \mathrm{ha}^{-1}$ in Y-MZ2, and $206 \mathrm{~kg} \mathrm{~N} \mathrm{ha}^{-1}$ in Y-MZ3. The plots receiving the same $\mathrm{N}$ rate of the whole field (N2; $144 \mathrm{~kg} \mathrm{~N} \mathrm{ha}^{-1}$ ) led to the following average yields: 2.2, 7.8, and $8.9 \mathrm{t} \mathrm{ha}^{-1}$ in Y-MZ1, Y-MZ2, 
and Y-MZ3, respectively. The apparent recovery of applied N averaged 29\% in Y-MZ1 and 68\% in Y-MZ2 and Y-MZ3.

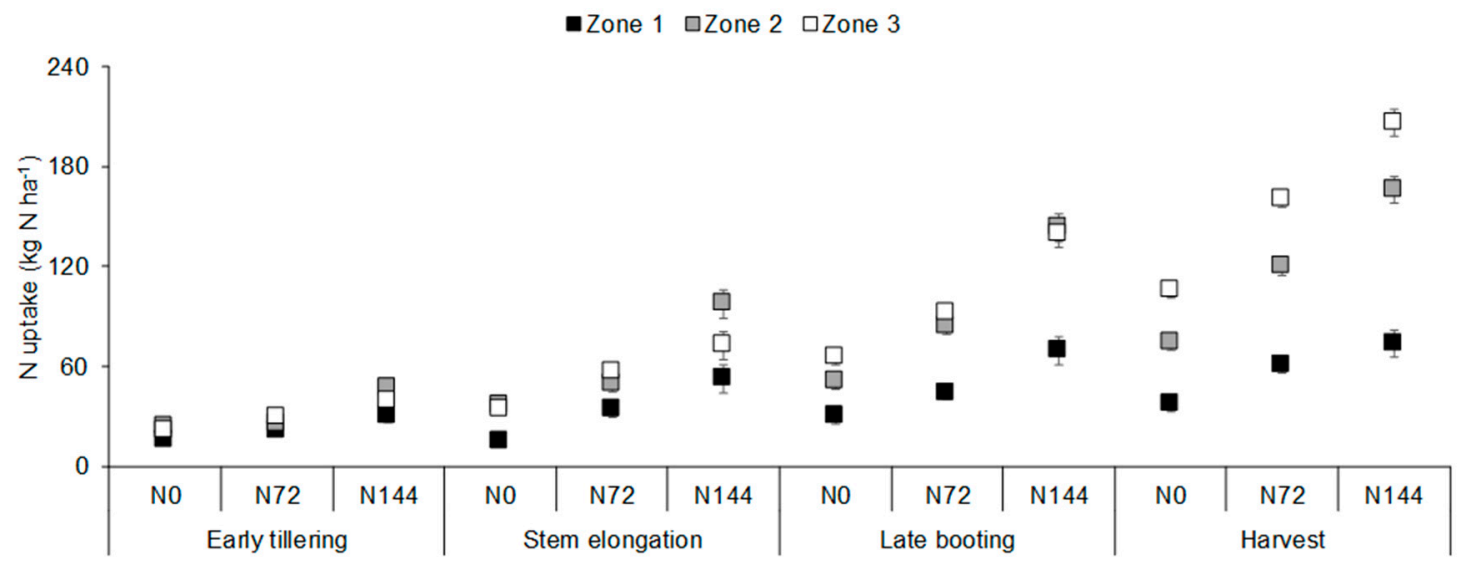

Figure 6. Mean $\mathrm{N}$ uptake values from the field trial presented separately by the time of monitoring survey, by nitrogen level ( $\mathrm{N} 0=0 \mathrm{~kg} \mathrm{~N} \mathrm{ha}^{-1} ; \mathrm{N} 72=72 \mathrm{~kg} \mathrm{~N} \mathrm{ha}^{-1} ; \mathrm{N} 144=144 \mathrm{~kg} \mathrm{~N} \mathrm{ha}^{-1}$ ) and by field zone (1, 2, and 3 representatives of low MZ1, medium MZ2, and high MZ3 grain yield, respectively). Error bars represent the standard error of the mean.

\section{Discussion}

\subsection{Multivariate Analysis}

The results showed that Y-MZ1 was the most recognizable area according to any source of information. The clustering carried out on the principal components confirmed that the largest contributors were the lowest ECa values and high reflectance values in the red region of the spectrum, irrespective of the monitoring stage. On the other hand, the relative size of Y-MZ2 and Y-MZ3 differed among development stages. Nonetheless, cluster analysis allowed discovering that NIR and red-edge reflectance values played a major contribution to the definition of Y-MZ2 and Y-MZ3 (Table S2). Our results confirmed the robustness of the red-edge index (NDRE) in the identification of crop status and consequently of MZs, in agreement with previous studies [29,30]. On the contrary, ECa played a minor role in the identification of Y-MZ2 and Y-MZ3 confirming that soil properties were not the only drivers of within-field variability. The crop spectral properties confirmed to be more informative for MZs identification at late wheat development [31,32], especially considering Y-MZ2 and Y-MZ3, even if they were statistically different for $\mathrm{N}$ uptake only at harvest. Spectral data acquired during the season allowed differentiating zones with less pronounced agronomic differences, that otherwise would have been uniformly managed.

\subsection{Soil-Based MZs Map}

The soil-MZs map obtained the highest portion of correct classification of Y-MZ1 (PCC= 0.77), indicating distinct soil properties with respect to the other field zones. The soil texture in Y-MZ1 (lowest yield) was sandy loam (sand $=77 \%$, clay $=11 \%$ ), whereas in Y-MZ2 and Y-MZ3 was loam (sand $=46 \%$, clay $=17 \%$ ) and sandy loam (sand $=55 \%$, clay $=14 \%$ ), respectively. In addition, Y-MZ1 had a lower organic matter content (1.0\%) compared to $1.4 \%$ and $1.7 \%$ measured in Y-MZ2 and Y-MZ3, respectively. The apparent recovery of N in Y-MZ1 was very low (29\%), suggesting high $\mathrm{N}$ losses or $\mathrm{N}$ accumulation in soil, whereas in Y-MZ2 and Y-MZ3, it was in line with previous experiments conducted under similar pedoclimatic conditions [33]. The Y-MZ1 was characterized by limited increases in AGB and N uptake in response to $\mathrm{N}$ fertilization $\left(<75 \mathrm{~kg} \mathrm{~N} \mathrm{ha}^{-1}\right)$. Therefore, the main yield-limiting factors in Y-MZ1 (Figure 6) were probably related to the low soil N supply to the crop, the high risk of nitrate leaching due to a coarse texture, and the low soil water content, in turn leading to water stress and 
reduced crop $\mathrm{N}$ availability. The low $\mathrm{N}$ availability, likely due to low soil $\mathrm{N}$ supply and late winter leaching (Figure S2), could have affected crop growth in Y-MZ1, in addition to water stress during April (stem elongation and booting), when precipitations were scarce (Figure S2).

This study confirmed that the sole consideration of soil properties in MZs mapping is not sufficient [32] and it could rather have led to the erroneous definition of Y-MZ2 and Y-MZ3. The difference in yield between Y-MZ2 and Y-MZ3 was probably caused by the interaction of soil properties with agricultural management factors, not accounted by soil survey. This is proved by the fact that soil-MZs classified correctly only $25 \%$ of Y-MZ3 with marked yield underestimation (BIAS $=0.57$ ). Moreover, the soil-MZs map showed large spatial variability within the field that was not reflected by Y-MZs (Figure 4). This suggested that the effect of soil properties on grain yield was partially masked by management and meteorological factors, in agreement with other studies $[8,34]$.

\subsection{Vegetation-Based MZs Map}

According to literature, the effects of local agronomic management and weather variability can be captured by vegetation indices [32], which in our study were more reliable than soil data for in-season MZs definition. The accuracy of VI-MZs maps increased along with wheat development, confirming that in-season monitoring allowed capturing the dynamics of crop growth, up to yield formation [9]. However, despite the site-specific accuracy of the VI-MZs maps increased, also the yield overestimation increased (Figure 5). This indicates a saturation effect common to all the vegetation indices, which occurs when vegetation is still growing while the indices had already reached their maximum value [35]. This situation emerged since StEl in large areas of the field (35\%,20\%, and $23 \%$ of the total field area for NDVI, GNDVI, and NDRE, respectively), which were erroneously transitioned from Y-MZ2 to Y-MZ3 because of high values of the vegetation indices. According to literature, our results confirmed that the red-based vegetation index (NDVI) was more affected by saturation than GNDVI and NDRE [36].

The cumulative vegetation indices probably suffered by saturation effect at earlier crop monitoring and did not lead to better results than the simple indices, according to Wang et al. (2014) [37] but in contrast to other studies $[25,38]$. It could be explained by the less sensitivity to crop growth dynamics due to a carry-over effect of the information of previous surveys [25]. One of the main evidence of our study is that AGB values and the crop $\mathrm{N}$ response were statistically different among Y-MZs from late booting, whereas vegetation indices were able to correctly reproduce Y-MZs since early wheat development. As a consequence, a minimum dataset for reliable MZs map definition can be defined as one vegetation index (RE-based, G-based, and even R-based at very early crop stages) collected between early tillering and stem elongation, which are also two key stages for wheat $\mathrm{N}$ side-dress fertilization.

\section{Conclusions}

Italian farmers are experiencing a transition from standard to precision agriculture and need simple and low-input procedures to support the shift of paradigm. This study evaluated the reliability of alternative data sources acquired by high-resolution soil and crop sensors when no past knowledge of the within-field variability is available. We demonstrated that soil-based maps were not able to reproduce the actual yield levels, whereas crop monitoring led to high accuracy, reliability, and discrimination ability in MZs mapping. The optimal source of information and timing of field survey were identified to allow a low-input and effective yield mapping of winter cereals: RE-based indices with a NIR component (coming from aerial or satellite surveys) collected from early tillering to stem elongation, which can be processed with simple statistical methods and open-source software could identify Y-MZs. Finally, despite the stability of MZs could be improved during the years with new yield maps, low-input, in-season crop monitoring demonstrated to be able to effectively support farmer management since early crop stages identifying MZs that could be effectively managed with different $\mathrm{N}$ rates. In fact, from an agronomical point of view, the identification of Y-MZ1, using the proposed procedure integrated with the results of the plot trial for the evaluation of $\mathrm{N}$ rates, suggested to reduce $\mathrm{N}$ fertilization in Y-MZ1 by about $50 \%$ with clear economic and environmental benefits. 
However, identified Y-MZ2 and Y-MZ3 that produced close but statistically different yields (7 and $9 \mathrm{t} \mathrm{DM} \mathrm{ha}{ }^{-1}$, respectively) would require a long-term evaluation to clearly identify the possibility of adopting different fertilization strategies.

Supplementary Materials: The following are available online at http://www.mdpi.com/2073-4395/10/8/1124/s1, Figure S1: Minimum (a) and maximum (b) daily temperature of seasons 2015 and 2016 compared with climatology of the period (years 1993-2016). Mean values (m), mean plus one standard deviation (m+1SD), mean plus two standard deviations $(\mathrm{m}+2 \mathrm{SD})$, mean minus one standard deviation $(\mathrm{m}-1 \mathrm{SD})$, mean minus two standard deviations (m-2SD) are shown. Figure S2: Monthly precipitation of seasons 2015 and 2016 compared with climatology of the period (years 1993-2016). Figure S3: Trial plots in the field situated in three zones identified through the visually inspection of Google RGB images (low, medium, and high crop vigor) and the preliminary analysis of soil ECa map (low, medium, and high). Zone 1 was characterized by low ECa and vigor, zone 2 by medium ECa and vigor, and zone 3 by high ECa and vigor. Figure S4: ECa map (mS m-1) acquired at $15 \mathrm{kHz}$ frequency. Figure S5: Mean aboveground biomass values from the plot experiment presented separately by time of monitoring survey, by nitrogen level (N0 $=0 \mathrm{~kg} \mathrm{~N} \mathrm{ha}^{-1} ; \mathrm{N} 72=0 \mathrm{~kg} \mathrm{~N} \mathrm{ha}^{-1} ; \mathrm{N} 144=144 \mathrm{~kg} \mathrm{~N} \mathrm{ha}^{-1}$ ) and by field zone $(1,2$, and 3 representative of low, medium, and high grain yield, respectively). Error bars represent the standard error of the mean. Table S1: Characterization of the clusters indicating for each cluster: the categorical variables (development stage, DVS: early tillering "eTl", stem elongation "StEl", late booting "1B"; the yield-based MZs corresponding to the yield levels 1 "low", 2 "medium", and 3 "high"); the percentage of pixels for each category of the categorical variables (Cla/Mod); the percentage of pixels classified with the indicated categorical variable (Mod/Cad); the percentage of pixels of all dataset classified with the indicated categorical variable (Global), the $p$-value $(\mathrm{P})$ of the student $\mathrm{t}$ test used to compare the average of the category with the general average; v.test (the transformation of $\mathrm{P}$ into a normal quantile). Categories are ordered by v.test values. Table S2: v.test results for each cluster. The mean and the standard deviation values for each "original variable" used for PCA analysis ("Mean in category" and "SD in category") are reported. Overall mean and standard deviation are also reported. Table S3: Results of the split-plot ANOVA for wheat above ground biomass at harvest ( $t$ dry matter $\mathrm{ha}^{-1}$ ), separately for each field survey. Significance of the differences between zones, the linear and quadratic trends of above ground biomass to $\mathrm{N}$ fertilizer, and their interactions are also reported. Table S4: Results of the split-plot ANOVA for wheat $\mathrm{N}$ uptake at harvest $\left(\mathrm{kg} \mathrm{N} \mathrm{ha}^{-1}\right)$, separately for each field survey. Significance of the differences between zones, the linear and quadratic trends of $\mathrm{N}$ uptake to $\mathrm{N}$ fertilizer, and their interactions are also reported.

Author Contributions: Conceptualization, M.C., P.M.G., D.C., B.O., G.C. (Giovanni Cabassi) and S.B.; Data curation, M.C., P.M.G., D.C., B.O. and S.B.; Formal analysis, M.C., D.C. and S.B.; Funding acquisition, P.M.G. and S.B.; Investigation, M.C., P.M.G., D.C., B.O., A.V.; Methodology, M.C., D.C. and S.B.; Project administration, P.M.G., S.B.; Resources, P.M.G., B.O., A.V., L.D. and S.B.; Supervision, P.M.G., D.C., G.C. (Giovanni Cabassi) and S.B.; Visualization, M.C., D.C. and G.C. (Gabriele Cola); Writing-original draft, M.C.; Writing-review \& editing, M.C., D.C., B.O., G.C. (Giovanni Cabassi) and S.B. All authors have read and agreed to the published version of the manuscript.

Funding: This research received no external funding.

Conflicts of Interest: The authors declare no conflict of interest.

\section{References}

1. Ramankutty, N.; Mehrabi, Z.; Waha, K.; Jarvis, L.; Kremen, C.; Herrero, M.; Rieseberg, L.H. Trends in global agricultural land use: Implications for environmental health and food security. Annu. Rev. Plant Biol. 2018, 69, 789-815. [CrossRef] [PubMed]

2. Taylor, J.; Whelan, B. A General Introduction to Precision Agriculture; Australian Center for Precision Agriculture. 2005. Available online: http://www.agriprecisione.it/wp-content/uploads/2010/11/general_ introduction_to_precision_agriculture.pdf (accessed on 29 July 2020).

3. Lemaire, G.; Jeuffroy, M.-H.; Gastal, F. Diagnosis tool for plant and crop N status in vegetative stage: Theory and practices for crop N management. Eur. J. Agron. 2008, 28, 614-624. [CrossRef]

4. Grisso, R.D.; Alley, M.M.; Thomason, W.E.; Holshouser, D.L.; Roberson, G.T. Precision Farming Tools: Variable-Rate Application. 2011. Available online: https:/vtechworks.lib.vt.edu/bitstream/handle/10919/ 47448/442-505_PDF.pdf (accessed on 29 July 2020).

5. Holland, K.H.; Schepers, J.S. Derivation of a variable rate nitrogen application model for in-season fertilization of corn. Agron. J. 2010, 102, 1415-1424. [CrossRef]

6. Solie, J.B.; Monroe, A.D.; Raun, W.R.; Stone, M.L. Generalized algorithm for variable-rate nitrogen application in cereal grains. Agron. J. 2012, 104, 378-387. [CrossRef] 
7. Dammer, K.-H.; Wartenberg, G. Sensor-based weed detection and application of variable herbicide rates in real time. Crop. Prot. 2007, 26, 270-277. [CrossRef]

8. Nawar, S.; Corstanje, R.; Halcro, G.; Mulla, D.; Mouazen, A.M. Delineation of soil management zones for variable-rate fertilization. Adv. Agron. 2017, 143, 175-245. [CrossRef]

9. Long, D.S.; Carlson, G.R.; De Gloria, S.D. Quality of field management maps. Site Specif. Manag. Agric. Syst. 1995, 251-271. [CrossRef]

10. Mulla, D.J. Using geostatistics and GIS to manage spatial patterns in soil fertility. In Automated Agriculture for the 21st Century; Kranzler, G., Ed.; ASAE: Niles, MI, USA, 1991; pp. 336-345.

11. Stewart, C.M.; McBratney, A.B.; Skerritt, J.H. Site-specific durum wheat quality and its relationship to soil properties in a single field in northern New South Wales. Precis. Agric. 2002, 3, 155-168. [CrossRef]

12. Shaner, D.L.; Khosla, R.; Brodahl, M.K.; Buchleiter, G.W.; Farahani, H.J. How well does zone sampling based on soil electrical conductivity maps represent soil variability? Agron. J. 2008, 100, 1472-1480. [CrossRef]

13. Schenatto, K.; Souza, E.G.; Bazzi, C.L.; Bier, V.A.; Betzek, N.M.; Gavioli, A. Data interpolation in the definition of management zones. Acta Sci. Technol. 2016, 38, 31-40. [CrossRef]

14. Casa, R.; Pelosi, F.; Pascucci, S.; Fontana, F.; Castaldi, F.; Pignatti, S.; Pepe, M. Early stage variable rate nitrogen fertilization of silage maize driven by multi-temporal clustering of archive satellite data. Adv. Anim. Biosci. 2017, 8, 288-292. [CrossRef]

15. Ortuani, B.; Sona, G.; Ronchetti, G.; Mayer, A.; Facchi, A. Integrating geophysical and multispectral data to delineate homogeneous management zones within a vineyard in Northern Italy. Sensors 2019, $19,3974$. [CrossRef] [PubMed]

16. Rampant, P.; Abuzar, M. Geophysical tools and digital elevation models: Tools for understanding crop yield and soil variability. Proceedings of SuperSoil 2004: 3rd Australian New Zealand Soils Conference, Sydney, Australia, 5-9 December 2004; University of Sydney: Sydney, Australia, 2004; pp. 1-9.

17. Pascucci, S.; Carfora, M.; Palombo, A.; Pignatti, S.; Casa, R.; Pepe, M.; Castaldi, F. A comparison between standard and functional clustering methodologies: Application to agricultural fields for yield pattern assessment. Remote Sens. 2018, 10, 585. [CrossRef]

18. De Benedetto, D.; Castrignano, A.; Diacono, M.; Rinaldi, M.; Ruggieri, S.; Tamborrino, R. Field partition by proximal and remote sensing data fusion. Biosyst. Eng. 2013, 114, 372-383. [CrossRef]

19. Khosla, R.; Inman, D.; Westfall, D.G.; Reich, R.M.; Frasier, M.; Mzuku, M.; Koch, B.; Hornung, A. A synthesis of multi-disciplinary research in precision agriculture: Site-specific management zones in the semi-arid western Great Plains of the USA. Precis. Agric. 2008, 9, 85-100. [CrossRef]

20. Guastaferro, F.; Castrignanò, A.; De Benedetto, D.; Sollitto, D.; Troccoli, A.; Cafarelli, B. A comparison of different algorithms for the delineation of management zones. Precis. Agric. 2010, 11, 600-620. [CrossRef]

21. Lancashire, P.D.; Bleiholder, H.; Boom, T.; van den Langelüddeke, P.; Stauss, R.; Weber, E.; Witzenberger, A. A uniform decimal code for growth stages of crops and weeds. Ann. Appl. Biol. 1991, 119, 561-601. [CrossRef]

22. Pebesma, E.; Heuvelink, G. Spatio-temporal interpolation using gstat. RFID J. 2016, 8, 204-218.

23. Ricotta, C.; Avena, G.; De Palma, A. Mapping and monitoring net primary productivity with AVHRR NDVI time-series: Statistical equivalence of cumulative vegetation indices. ISPRS J. Photogramm. Remote Sens. 1999, 54, 325-331. [CrossRef]

24. Lê, S.; Josse, J.; Husson, F. FactoMineR: An R package for multivariate analysis. J. Stat. Softw. 2008, 25, 1-18. [CrossRef]

25. R Development Core Team. R: A Language and Environment for Statistical Computing; R Foundation for Statistical Computing: Vienna, Austria, 2008; ISBN 3-900051-07-0. Available online: http://www.r-project.org (accessed on 29 July 2020).

26. Fridgen, J.J.; Kitchen, N.R.; Sudduth, K.A.; Drummond, S.T.; Wiebold, W.J.; Fraisse, C.W. Management zone analyst (MZA). Agron. J. 2004, 96, 100-108. [CrossRef]

27. Martínez-Casasnovas, J.A.; Arnó, J. Use of farmer knowledge in the delineation of potential management zones in precision agriculture: A case study in maize (Zea mays L.). Agriculture 2018, 8, 84.

28. Wilks, D.S. Statistical Methods in the Atmospheric Sciences; Elsevier: Amsterdam, The Netherlands; Academic Press: Amsterdam, The Netherlands, 2011.

29. Tavakoli, H.; Mohtasebi, S.S.; Alimardani, R.; Gebbers, R. Evaluation of different sensing approaches concerning to non-destructive estimation of leaf area index (LAI) for winter wheat. Int. J. Smart Sens. Intell. Syst. 2014, 7, 337-359. 
30. Corti, M.; Cavalli, D.; Cabassi, G.; Gallina, P.M.; Bechini, L. Does remote and proximal optical sensing successfully estimate maize variables? A review. Eur. J. Agron. 2018, 99, 37-50. [CrossRef]

31. Marino, S.; Alvino, A. Detection of homogeneous wheat areas using multi-temporal UAS images and ground truth data analyzed by cluster analysis. Eur. J. Remote Sens. 2018, 51, 266-275. [CrossRef]

32. Maestrini, B.; Basso, B. Predicting spatial patterns of within-field crop yield variability. Field Crop. Res. 2018, 219, 106-112. [CrossRef]

33. Cavalli, D.; Cabassi, G.; Borrelli, L.; Geromel, G.; Bechini, L.; Degano, L.; Marino Gallina, P. Nitrogen fertilizer replacement value of undigested liquid cattle manure and digestates. Eur. J. Agron. 2016, 73, 34-41. [CrossRef]

34. Schepers, A.R.; Shanahan, J.F.; Liebig, M.A.; Schepers, J.S.; Johnson, S.H.; Luchiari, A. Appropriateness of management zones for characterizing spatial variability of soil properties and irrigated corn yields across years. Agron. J. 2004, 96, 195-203. [CrossRef]

35. Xia, T.; Miao, Y.; Wu, D.; Shao, H.; Khosla, R.; Mi, G. Active optical sensing of spring maize for in-season diagnosis of nitrogen status based on nitrogen nutrition index. Remote Sens. 2016, 8, 605. [CrossRef]

36. Scotford, I.M.; Miller, P.C.H. Applications of spectral reflectance techniques in Northern European cereal production: A review. Biosyst. Eng. 2005, 90, 235-250. [CrossRef]

37. Wang, L.; Tian, Y.; Yao, X.; Zhu, Y.; Cao, W. Predicting grain yield and protein content in wheat by fusing multi-sensor and multi-temporal remote-sensing images. Field Crop. Res. 2014, 164, 178-188. [CrossRef]

38. Zhou, X.; Zheng, H.B.; Xu, X.Q.; He, J.Y.; Ge, X.K.; Yao, X.; Cheng, T.; Zhu, Y.; Cao, W.X.; Tian, Y.C. Predicting grain yield in rice using multi-temporal vegetation indices from UAV-based multispectral and digital imagery. ISPRS J. Photogramm. Remote Sens. 2017, 130, 246-255. [CrossRef]

(C) 2020 by the authors. Licensee MDPI, Basel, Switzerland. This article is an open access article distributed under the terms and conditions of the Creative Commons Attribution (CC BY) license (http://creativecommons.org/licenses/by/4.0/). 